\title{
Differential Equations and Their Applications
}




\section{Martin Braun}

\section{Differential Equations and Their Applications}

(Third Edition) Short Version

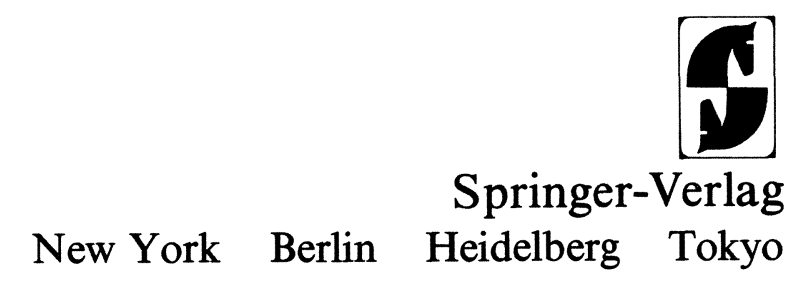




\section{Martin Braun}

Department of Mathematics

Queens College

City University of New York

Flushing, NY 11367

USA

AMS Subject Classification: 34-01

Library of Congress Cataloging in Publication Data

Braun, Martin, 1941-

Differential equations and their applications.

Abridged version of: Differential equations and their applications. 3rd ed. c1983.

Includes index.

1. Differential equations. I. Title.

QA371.B7932 $1983 \quad 515.3^{\prime} 5 \quad 83-4760$

All rights reserved.

No part of this book may be translated or reproduced in any form without written permission from Springer-Verlag, 175 Fifth Avenue,

New York, New York 10010, USA.

(C) 1983 by Springer-Verlag New York Inc.

Softcover reprint of the hardcover 1st edition 1983

This book is an abridged version of Differential Equations and Their Applications:

An Introduction to Applied Mathematics, 3rd ed., by Martin Braun, (C) 1983 by Springer-Verlag New York Inc.

$\begin{array}{lllllllll}9 & 8 & 7 & 6 & 5 & 4 & 3 & 2 & 1\end{array}$

ISBN-13: 978-1-4684-0175-2

e-ISBN-13: 978-1-4684-0173-8

DOI: $10.1007 / 978-1-4684-0173-8$ 
To four beautiful people:

Zelda Lee

Adeena Rachelle, I. Nasanayl, and Shulamit 


\section{Preface to the Third Edition}

There are two major changes in the Third Edition of Differential Equations and Their Applications. First, we have completely rewritten the section on singular solutions of differential equations. A new section, 2.8.1, dealing with Euler equations has been added, and this section is used to motivate a greatly expanded treatment of singular equations in sections 2.8.2 and 2.8.3.

Our second major change is in Section 2.6, where we have switched to the metric system of units. This change was requested by many of our readers.

In addition to the above changes, we have updated the material on population models, and have revised the exercises in this section. Minor editorial changes have also been made throughout the text. 


\section{Preface to the First Edition}

This textbook is a unique blend of the theory of differential equations and their exciting application to "real world" problems. First, and foremost, it is a rigorous study of ordinary differential equations and can be fully understood by anyone who has completed one year of calculus. However, in addition to the traditional applications, it also contains many exciting "real life" problems. These applications are completely self contained. First, the problem to be solved is outlined clearly, and one or more differential equations are derived as a model for this problem. These equations are then solved, and the results are compared with real world data. The following applications are covered in this text.

1. In Section 1.3 we prove that the beautiful painting "Disciples at Emmaus" which was bought by the Rembrandt Society of Belgium for $\$ 170,000$ was a modern forgery.

2. In Section 1.5 we derive differential equations which govern the population growth of various species, and compare the results predicted by our models with the known values of the populations.

3. In Section 1.6 we try to determine whether tightly sealed drums filled with concentrated waste material will crack upon impact with the ocean floor. In this section we also describe several tricks for obtaining information about solutions of a differential equation that cannot be solved explicitly.

4. In Section 2.7 we derive a very simple model of the blood glucose regulatory system and obtain a fairly reliable criterion for the diagnosis of diabetes.

5. In Section 4.3 we derive two Lanchestrian combat models, and fit one of these models, with astonishing accuracy, to the battle of Iwo Jima in World War II. 
This textbook also contains the following important, and often unique features.

1. In Section 1.9 we give a complete proof of the existence-uniqueness theorem for solutions of first-order equations. Our proof is based on the method of Picard iterates, and can be fully understood by anyone who has completed one year of calculus.

2. Modesty aside, Section 2.12 contains an absolutely super and unique treatment of the Dirac delta function. We are very proud of this section because it eliminates all the ambiguities which are inherent in the traditional exposition of this topic.

3. All the linear algebra pertinent to the study of systems of equations is presented in Sections 3.1-3.5. One advantage of our approach is that the reader gets a concrete feeling for the very important but extremely abstract properties of linear independence, spanning, and dimension. Indeed, many linear algebra students sit in on our course to find out what's really going on in their course.

I greatly appreciate the help of the following people in the preparation of this manuscript: Eleanor Addison who drew the original figures, and Kate MacDougall, Sandra Spinacci, and Miriam Green who typed portions of this manuscript.

I am grateful to Walter Kaufmann-Bühler, the mathematics editor at Springer-Verlag, and Elizabeth Kaplan, the production editor, for their extensive assistance and courtesy during the preparation of this manuscript. It is a pleasure to work with these true professionals.

Finally, I am especially grateful to Joseph P. LaSalle for the encouragement and help he gave me. Thanks again, Joe. 


\section{Contents}

\section{Chapter 1}

First-order differential equations $\quad 1$

1.1 Introduction 1

1.2 First-order linear differential equations 2

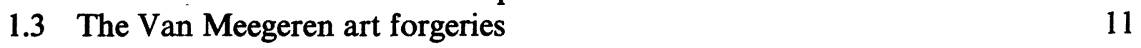

$\begin{array}{ll}1.4 & \text { Separable equations } \\ 1.5 & 20\end{array}$

$\begin{array}{ll}1.5 & \text { Population models } \\ 1.6 & 26\end{array}$

1.6 An atomic waste disposal problem $\quad 39$

1.7 The dynamics of tumor growth, mixing problems, and

1.8 Exact equations, and why we cannot solve very many $\begin{array}{ll}\text { differential equations } & 51\end{array}$

1.9 The existence-uniqueness theorem; Picard iteration 61

1.10 Difference equations, and how to compute the interest
due on your student loans

Chapter 2

Second-order linear differential equations $\quad 80$

$\begin{array}{ll}2.1 & \text { Algebraic properties of solutions } \\ 2.2 & 80\end{array}$

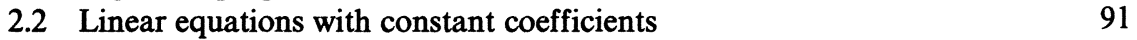

2.2.1 Complex roots 94

2.2.2 Equal roots; reduction of order 98

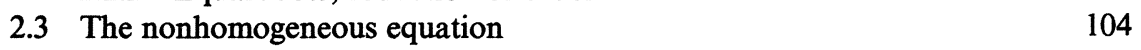

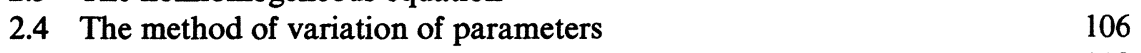

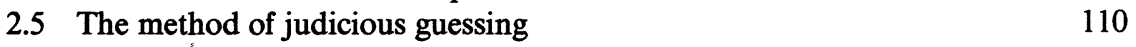

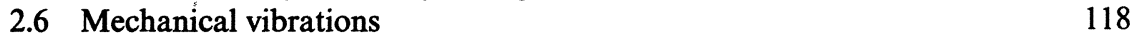

$\begin{array}{ll}\text { 2.6.1 The Tacoma Bridge disaster } & 126\end{array}$

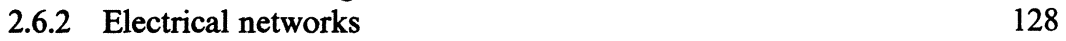


2.7 A model for the detection of diabetes 131

$\begin{array}{lll}2.8 & \text { Series solutions } & 138\end{array}$

$\begin{array}{ll}\text { 2.8.1 Singular points, Euler equations } & 150\end{array}$

2.8.2 Regular singular points, the method of Frobenius 155

2.8.3 Equal roots, and roots differing by an integer 171

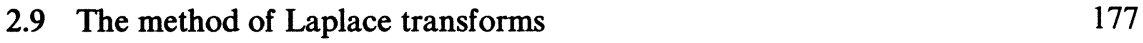

$\begin{array}{ll}2.10 \text { Some useful properties of Laplace transforms } & 185\end{array}$

$\begin{array}{ll}2.11 \text { Differential equations with discontinuous right-hand sides } & 190\end{array}$

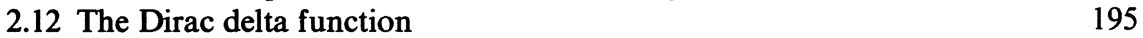

$\begin{array}{ll}2.13 \text { The convolution integral } & 203\end{array}$

2.14 The method of elimination for systems 209

$\begin{array}{ll}2.15 \text { Higher-order equations } & 211\end{array}$

Chapter 3

Systems of differential equations 216

3.1 Algebraic properties of solutions of linear systems 216

$\begin{array}{ll}3.2 & \text { Vector spaces } \\ 3.3 & 225\end{array}$

$\begin{array}{lll}\text { 3.3 Dimension of a vector space } & 231\end{array}$

3.4 Applications of linear algebra to differential equations 243

3.5 The theory of determinants 249

3.6 The eigenvalue-eigenvector method of finding solutions 262

$\begin{array}{ll}3.7 \text { Complex roots } & 271\end{array}$

$\begin{array}{lll}3.8 & \text { Equal roots } & 275\end{array}$

$\begin{array}{ll}\text { 3.9 Fundamental matrix solutions; } e^{\mathbf{A} t} & 285\end{array}$

3.10 The nonhomogeneous equation; variation of parameters 290

3.11 Solving systems by Laplace transforms 298

\section{Chapter 4}

Qualitative theory of differential equations 302

4.1 Introduction 302

4.2 The phase-plane 308

4.3 Lanchester's combat models and the battle of Iwo Jima 313

Appendix A

Some simple facts concerning functions

of several variables

Appendix B

Sequences and series $\quad 324$

Answers to odd-numbered exercises 326

Index 339 